\title{
Application of D-Dimer in Prognosis of Pulmonary Embolism
}

\author{
Urvashi Gupta ${ }^{1} \quad$ Jagannath S. Dhadwad ${ }^{1}$ Prasad A. Gurjar ${ }^{2} \quad$ Dineshsingh Patil $^{1}$ \\ ${ }^{1}$ Department of General Medicine, Dr. D. Y. Patil Medical College, \\ Hospital and Research Centre, Pune, Maharashtra, India \\ 2Department of Nephrology, Dr. D. Y. Patil Medical College, \\ Hospital and Research Centre, Pune, Maharashtra, India

\begin{abstract}
Address for correspondence Urvashi Gupta, Department of Research Centre, Pimpri, Pune 411018, Maharashtra, India (e-mail: urva.gupta12@gmail.com).
\end{abstract} \\ General Medicine, Dr. D. Y. Patil Medical College, Hospital and
}

Int J Recent Surg Med Sci 2019;5:15-18

\begin{abstract}
Introduction Pulmonary embolism (PE) is a relatively common cardiovascular emergency. It can lead to a variety of outcomes, from potentially reversible right ventricular failure (by occluding the pulmonary arterial bed) to eventual death. The diagnosis of PE is often missed due to its nonspecific clinical presentation.

Materials and Methods This is a descriptive cross-sectional study conducted at tertiary care center in India from October 1, 2016 to September 30, 2018, which

Keywords

- pulmonary arteries

- pulmonary embolism

- pulmonary embolism rule-out criteria

- blood sugar level

- computed tomography

- chest X-ray

- deep vein thrombosis

- electrocardiography

- high-density lipoprotein

- low-density lipoprotein

- venous

thromboembolism includes 36 cases of PE. Institutional ethics committee clearance was obtained before start of the study. Written and informed consent was obtained from all patients. Patients were informed regarding purpose, procedures, risks, and benefits of the study in their own vernacular language.

Results The age-wise distribution of patients in this study showed that most patients were up to 40 years amounting to $58.4 \%$ of cases. Sex-wise distribution also showed that most patients were male (66.7\%). Fifty percent of the patients had no thrombosis on Color Doppler findings of the involved limb, and $31.25 \%$ had superficial venous thrombosis. Blood investigation revealed that $94.4 \%$ patients had troponin negative and $94.4 \%$ had D-dimer positive. This study also attempted to correlate the D-dimer findings and Well's score. It can be seen that as per Well's score, 13 patients were with high probability, 18 with moderate probability, and 5 with low probability.

Conclusion This study states that D-dimer assays are highly sensitive and have a low rate of false-negative results. The assays have been found safe and effective when the clinical probability of PE is low. When the D-dimer result is negative in patients with low clinical probability of having PE, the presence of acute PE can be safely ruled out without diagnostic imaging.
\end{abstract}

\section{Introduction}

Pulmonary embolism (PE) is a relatively common cardiovascular emergency. It can lead to a variety of outcomes, from potentially reversible right ventricular failure (by occluding the pulmonary arterial bed) to eventual death. The diagnosis of PE is often missed due to its nonspecific clinical presentation. Early initiation of therapy is very beneficial if detected in time. Initial therapy is primarily aimed at lifesaving restoration of flow through the occluded pulmonary arteries (PAs) and at preventing fatality. However,

\section{received}

February 9, 2019

accepted

February 19, 2019
DOI https://doi.org/

10.1055/s-0039-1688543

ISSN 2455-7420. it is imperative to justify initial treatment and long-term anticoagulation necessary for secondary prevention in each patient by means of a validated diagnostic strategy. ${ }^{1}$

Most patients succumb to death due to failure to reach the diagnosis of PE rather than inadequate treatment because PE often occurs without any previous predisposing factors. The diagnosis of PE requires high degree of suspicion. As predisposing factors required to exclude or confirm PE are both nonspecific and nonconfirmatory on their own, noninvasive imaging techniques are the backbone of diagnosis. Diagnostic strategies for the evaluation
License terms Update Society 
of PE have changed and evolved tremendously over the years. ${ }^{2}$ Technical advances in speed and spatial resolution have allowed computed tomographic (CT) angiography to be used in vascular imaging. D-dimer antibodies have high specificity for venous thromboembolism (VTE). As opposed to other markers that can only detect products of acute coagulation, D-dimer assays expand the diagnostic window. $^{3}$

This study was an attempt to correlate between clinical profiles of PE patients with special reference to D-dimer to quickly identify patients of PE.

Also, pulmonary embolism rule-out criteria (PERC) can be used in patients presenting in emergency department and suspected of acute PE. It helps rule out risk of PE in people when they have already been stratified into a lowrisk category according to modified Well's score and avoids unnecessary further investigations. ${ }^{4}$

\section{Materials and Methods}

A descriptive cross-sectional study was undertaken at a tertiary care center in India to study the correlation between clinical profile and D-dimer for assessment of PE. A total of 36 patients suspected of having PE constituted this study.

The exclusion criteria were

1. Age $<12$ years.

2. Patients in whom CT pulmonary angiography was contraindicated such as pregnancy, allergies to intravenous contrast, deranged renal function tests, and hemodynamically unstable or cardiac issues.

A detailed history and clinical examination was performed on patients having PE with special emphasis on the history of PE or deep vein thrombosis (DVT), any injury that damages veins, having catheter placed in the vein, taking birth control pills or undergoing hormonal therapy, smoking, active cancer (treatment ongoing or within previous 6 months of palliative treatment), paralysis, paresis, bedridden for $>3$ days or major surgery within 4 weeks, etc. D-dimer was done in all suspected patients with moderate to high pretest probability and in those who do not fulfill PERC with low pretest probability.

The data were entered in Microsoft Excel and analyzed using Epi 7. Qualitative data were summarized using proportions and percentages and quantitative data with mean and standard deviation. Appropriate test of statistical significance such as analysis of variance (ANOVA) was used to analyze, with $p<0.05$ as significant value at $95 \%$ confidence interval.

\section{Results}

The age-wise distribution of patients in this study showed that most patients were up to 40 years amounting to $58.4 \%$ of cases. Sex-wise distribution also showed that most patients were male (66.7\%). The chief complaints shown by the patients are presented in - Table 1. It is evident that patients showed dyspnea, cough with or without expectoration, fever, hemoptysis, chest pain, and palpitation as their presenting complaints.
Patients were subjected to modified Well's score probability, and it was recorded that most patients were in moderate probability level (50\%).

$\mathrm{X}$-ray findings in this study population showed that most patients had normal chest X-ray (44\%), followed by consolidation (19.4\%), costophrenic (CP) angle blunting (16.7\%), opacity (11.1\%), and other findings were found in only few cases (- Table 2 ).

Patients were subjected to assessment of troponin levels, and it was observed that $94.4 \%$ cases were positive. Similar results were also shown by D-dimer analysis ( - Table 3 ).

This study also attempted to correlate the D-dimer findings and Well's score. The details are presented in - Table 4. It can be seen that as per Well's score, 13 patients were with high probability, 18 with moderate probability, and 5 with low probability.

Table 1 Sex-wise distribution, major complaints, and continuous variable findings among patients

\begin{tabular}{|c|c|c|}
\hline & Frequency & Percent \\
\hline \multicolumn{3}{|l|}{ Sex } \\
\hline Female & 12 & 33.3 \\
\hline Male & 24 & 66.7 \\
\hline Total & 36 & 100 \\
\hline \multicolumn{3}{|l|}{ Complaints } \\
\hline Dyspnea & 34 & 94.4 \\
\hline Cough with expectoration & 14 & 38.9 \\
\hline Variables & Mean & SD \\
\hline Age (y) & 46.5 & 18.5 \\
\hline $\mathrm{SpO}_{2}$ at room air (\%) & 89.4 & 4.7 \\
\hline Modified Well's score & 5.5 & 2.4 \\
\hline D-dimer & $1,441.6$ & $1,184.7$ \\
\hline
\end{tabular}

Table 2 Chest $X$-ray findings among patients

\begin{tabular}{|l|l|l|}
\hline Chest X-ray & Frequency & Percentage \\
\hline Normal & 16 & 44.4 \\
\hline Consolidation & 7 & 19.4 \\
\hline Costophrenic (CP) blunting & 6 & 16.7 \\
\hline Opacity & 4 & 11.1 \\
\hline Palla's sign & 2 & 5.6 \\
\hline Infiltrate & 1 & 2.8 \\
\hline Total & 36 & 100 \\
\hline
\end{tabular}

Table 3 Troponin and D-dimer findings in patients

\begin{tabular}{|c|l|l|}
\hline \multicolumn{2}{|l|}{ Frequency } & Percentage \\
\hline \multicolumn{2}{|l|}{ Troponin } \\
\hline Negative & 34 & 94.4 \\
\hline Positive & 2 & 5.6 \\
\hline D-dimer & 94.4 \\
\hline Positive & 34 & 5.6 \\
\hline Negative & 2 &
\end{tabular}


Table 4 D-dimer and Well's score correlation

\begin{tabular}{|l|l|l|l|l|}
\hline Well's score & Mean & Standard deviation & Minimum & Maximum \\
\hline High probability $(n=13)$ & $1,057.7$ & 663.68 & 100 & 2,430 \\
\hline Moderate probability $(n=18)$ & $1,366.0$ & $1,486.85$ & 0 & 5,720 \\
\hline Low probability $(n=5)$ & $1,847.2$ & $1,165.79$ & 460 & 3,200 \\
\hline Total $(n=36)$ & $1,321.5$ & $1,202.7$ & 0 & 5,720 \\
\hline
\end{tabular}

\section{Discussion}

PE encompasses a gradient of clinical presentations, ranging from asymptomatic disease to life-threatening massive PE manifesting as rapidly evolving dangerous hypotension and cardiogenic shock. There are hardly any published data on PE from India. Due to lack of adequate specificity, the clinical presentation and investigations alone cannot be relied on to confirm or rule out PE. ${ }^{5} \mathrm{~A}$ number of factors have been assigned as the cause of PE such as hypercoagulable states, diabetes mellitus, most recent surgery, heart failure, hypertension, smoking, dyslipidemia, etc. ${ }^{6,7}$ Similar results were also obtained in this study in which the major complaints observed were dyspnea, cough with or without expectoration, fever, hemoptysis, chest pain, and palpitation. It has further been documented that the patients who died unexpectedly from PE, complained of nagging symptoms, often for weeks before dying.?

Pretest probability scores along with clinical suspicion aid in early diagnosis of high-risk patients with acute PE. ${ }^{8}$ In the past, various techniques were used to diagnose PE, which included chest X-ray, electrocardiogram (ECG), CT angiography, etc. However, all these techniques have limitations. This study assessed the use of D-dimer for ascertaining PE.

D-dimers are cross-linked degradation products of fibrin, and their presence in human plasma is an indicator of fibrinolytic activity, ${ }^{9}$ which is a sign of acute thromboembolic disease. ${ }^{10} \mathrm{D}$-dimer assays are highly sensitive and have a low rate of false-negative results. The assays have been found safe and effective when the clinical probability of PE is low. ${ }^{11} \mathrm{D}$-dimer levels correlate with the presence of fibrin clots without regard to location. In a variety of other medical conditions such as trauma, recent surgery, hemorrhage, cancer, and sepsis that are associated with activation of the coagulation system and formation of fibrin clots, D-dimer levels are elevated. ${ }^{12}$ This explains the low specificity of the D-dimer assay for VTE. Patients with a likely clinical probability should undergo further testing, regardless of the D-dimer test outcome. Therefore, after assessing patient's clinical probability, D-dimer concentration should also be done to prevent from being misguided by a normal D-dimer result even when the patient falls in the clinical probability. ${ }^{12}$ During this study, D-dimer was found to be positive in $94.4 \%$ cases (-Table 3 ) and correlated with Well's score. In view of the above, it was concluded that the D-dimer may be used as test for prognosis of PE in combination with clinical probability and CT pulmonary angiography that is considered as the gold standard for diagnosing a suspected case of PE. ${ }^{13}$

\section{Conclusion}

PE, though serious clinical problem, is often underrecognized and underdiagnosed all over the world. Diagnosis of PE is more difficult than treatment. A high index of suspicion is necessary to consider the diagnosis. Using modified Well's criteria helps stratify patients according to risk. D-dimer is a simple test that should be performed in all suspected patients. Whereas a negative result practically rules out thrombosis, a positive result can indicate thrombosis but does not rule out other potential causes. For a very high score, or pretest probability, a D-dimer will make a little difference and anticoagulant therapy will be initiated regardless of test results, and additional testing for DVT and PE may be performed. For a moderate or low score, or pretest probability, a negative D-dimer will virtually rule out thromboembolism, but if the D-dimer results are high, further testing is required to confirm the diagnosis. The increasing availability of newer imaging modalities such as CT pulmonary angiography is likely to improve its diagnosis. When diagnostic techniques are not immediately available, empiric treatment should be initiated anyway. Early recognition and aggressive and appropriate therapy improve the outcome in potentially fatal condition.

\section{Conflict of Interest}

None declared.

\section{References}

1 Roy PM, Meyer G, Vielle B, et al; EMDEPU Study Group. Appropriateness of diagnostic management and outcomes of suspected pulmonary embolism. Ann Intern Med 2006;144(3):157-164

2 Stein PD, Woodard PK, Hull RD, et al. Gadolinium-enhanced magnetic resonance angiography for detection of acute pulmonary embolism: an in-depth review. Chest 2003;124(6):2324-2328

3 Reber G, de Moerloose P. D-dimer assays for the exclusion of venous thromboembolism. Semin Thromb Hemost 2000;26(6):619-624

4 Sharif S, Kearon C, Eventov M, et al. P104: Evaluating the use of the pulmonary embolism rule-out criteria in the emergency department. CJEM 2018;20(S1):S93-S94

5 Fedullo PF, Tapson VF. Clinical practice. The evaluation of suspected pulmonary embolism. $N$ Engl J Med 2003;349(13):1247-1256

6 Algahtani FH, Bayoumi N, Abdelgadir A, et al. Clinical characteristics and risk factors of pulmonary embolism: data from a Saudi tertiary-care center. J Thromb Haemost 2013;11(2):388-390

7 Bilgehan D, Oguzturk H, Turtay MG, et al. Pulmonary embolism: single and multiple risk factors. Biomedical Research India 2017;28(9):4213-4218 
8 Dutton J, Dachsel M, Crane R. Can the use of an age-adjusted D-dimer cut-off value help in our diagnosis of suspected pulmonary embolism? Clin Med (Lond) 2018;18(4):293-296

9 Budzynski AZ, Marder VJ, Parker ME, Shames P, Brizuela BS, Olexa SA. Antigenic markers on fragment DD, a unique plasmic derivative of human crosslinked fibrin. Blood 1979;54(4):794-804

10 Wilson DB, Gard KM. Evaluation of an automated, latex-enhanced turbidimetric D-dimer test (advanced D-dimer) and usefulness in the exclusion of acute thromboembolic disease. Am J Clin Pathol 2003;120(6):930-937
11 van Belle A, Büller HR, Huisman MV, et al; Christopher Study Investigators. Effectiveness of managing suspected pulmonary embolism using an algorithm combining clinical probability, D-dimer testing, and computed tomography. JAMA 2006;295(2):172-179

12 Dhananjaya M, Meti K, Parakh R. D-dimer negative pulmonary embolism. International Journal of Advances in Medicine. 2018;5(2):429-432

13 Moore AJE, Wachsmann J, Chamarthy MR, Panjikaran L, Tanabe Y, Rajiah P. Imaging of acute pulmonary embolism: an update. Cardiovasc Diagn Ther 2018;8(3):225-243 